\title{
Cadmium substituted high permeability lithium ferrite
}

\author{
S S BELLAD, S C WATAWE, A M SHAIKH and B K CHOUGULE* \\ Department of Physics, Shivaji University, Kolhapur 416 004, India
}

MS received 5 April 1999; revised 17 January 2000

\begin{abstract}
Polycrystalline $\mathrm{Li}_{0.5-x / 2} \mathrm{Cd}_{x} \mathrm{Fe}_{2.5-x / 2} \mathrm{O}_{4}$ ferrites where $x=0,0.1,0.2,0.3,0.4,0.5$ and 0.6 were prepared by a double sintering ceramic technique and characterized by $\mathrm{X}$-ray diffraction and scanning electron microscopy (SEM). The lattice parameter is found to increase monotonically with the cadmium content. It is explained in terms of the sizes of component ions. The grain size of the samples increases up to $x=0 \cdot 3$ and then it decreases for higher values of $x$. A similar trend is observed in the variation of Ms with $\mathrm{Cd}^{2+}$ content. The initial permeability $\left(\mu_{\mathrm{i}}\right)$ is however found to increase continuously with $x$. The increase in $\mu_{\mathrm{i}}$ is attributed to decrease of anisotropy constant $K_{1}$ and higher grain size of the samples.
\end{abstract}

Keywords. Lithium ferrites; initial permeability; grain size; microstructure; magnetic properties.

\section{Introduction}

Lithium ferrite and mixed lithium ferrites have very high potential for microwave applications, especially as replacements for garnets, due to their low cost. The squareness of the hysteresis loop and superior temperature performance are other prominent advantages that have made them very promising candidates for application in microwave devices. In particular, mixed lithium-cadmium ferrites are of much interest because of their applications in microwave devices such as isolators, circulators, gyrators and phase shifters (Ravinder 1994). Several investigations on properties of mixed $\mathrm{Li}-\mathrm{Cd}$ ferrites, such as structural, electrical conductivity, thermoelectric power, elastic properties, frequency and composition dependence of dielectric behaviour, have been reported in the literature (Ravinder and Seshagiri Rao 1990; Radha and Ravinder 1995; Bellad and Chougule 1998). However, we found no such reports on the microstructure, magnetization and permeability behaviour of $\mathrm{Li}-\mathrm{Cd}$ ferrites. In this communication we report our studies on these parameters.

\section{Experimental}

The polycrystalline lithium-cadmium ferrite samples with the chemical formula $\mathrm{Li}_{0.5-x / 2} \mathrm{Cd}_{x} \mathrm{Fe}_{2.5-x / 2} \mathrm{O}_{4}$ (where $x=0$, $0 \cdot 1,0 \cdot 2,0 \cdot 3,0 \cdot 4,0 \cdot 5$, and $0 \cdot 6)$ were prepared by conventional ceramic technique using $\mathrm{AR}$ grade component oxides. Final sintering of the samples was carried out at $1000^{\circ} \mathrm{C}$ for $24 \mathrm{~h}$ to avoid the loss of lithium. Pellets

*Author for correspondence
( $1 \mathrm{~cm}$ dia. and $0.3 \mathrm{~cm}$ thick) and torroids ( $2 \mathrm{~cm}$ O.D. and $1 \mathrm{~cm}$ I.D.) pressed at 8 tonnes $/ \mathrm{cm}^{2}$ with a hydraulic press were used in the measurements of magnetization and initial permeability, respectively. Magnetization measurements were performed with a high field hysteresis loop tracer at a constant magnetic field of $3 \mathrm{kOe}$ and initial permeability measurements were performed with a LCR-Q meter (APLAB, model 4912) at a fixed frequency of $1 \mathrm{kHz}$. The microstructure was studied with a scanning electron microscope (Cambridge Stereoscan, $250 \mathrm{MK}$ III). The structure formation was confirmed by X-ray diffraction using $\mathrm{CuK} \alpha(1.5418 \AA)$ radiation.

\section{Results and discussion}

The single-phase spinel nature of the samples was confirmed from X-ray diffraction patterns. The lattice parameter increases linearly with cadmium content obeying the Vegard's law. The increase in lattice parameter is expected due to substitutional occupancy since the ionic radii of $\mathrm{Fe}^{3+}, \mathrm{Li}^{1+}$, and $\mathrm{Cd}^{2+}$ in tetrahedral and octahedral sites are in the order $0.065\left(\mathrm{Fe}^{3+}\right)<0.071\left(\mathrm{Li}^{1+}\right)<0.084$ $\left(\mathrm{Cd}^{2+}\right) \mathrm{nm}$ respectively (Bellad and Chougule 1998).

The variation of the mean grain diameter $\left(d_{\mathrm{m}}\right)$ obtained from SEM micrographs with content of cadmium is shown in figure 1. It is observed that the average grain diameter increases to a maximum for sample with $x=0 \cdot 3$ and then decreases slowly for further increase in $x$. This is due to the fact that the ions like $\mathrm{Cd}^{2+}, \mathrm{Zn}^{2+}, \mathrm{Ti}^{4+}$ do not favour grain growth when present in excess quantities (Patil et al 1991).

Figure 2 shows the variation of saturation magnetization with content of cadmium. The increase in magnetization with content of cadmium up to $x=0 \cdot 3$ is due to dilution of 


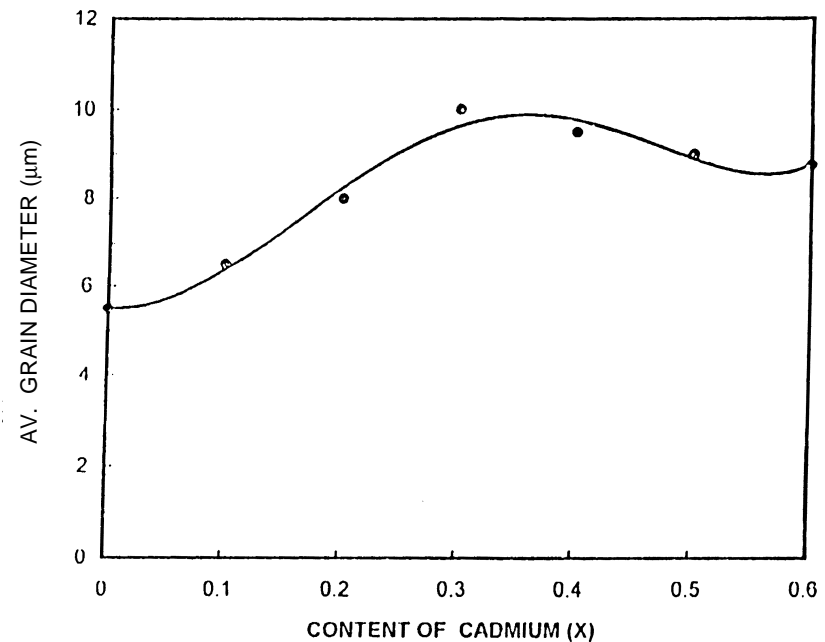

Figure 1. Variation of average grain diameter with content of cadmium $(x)$.

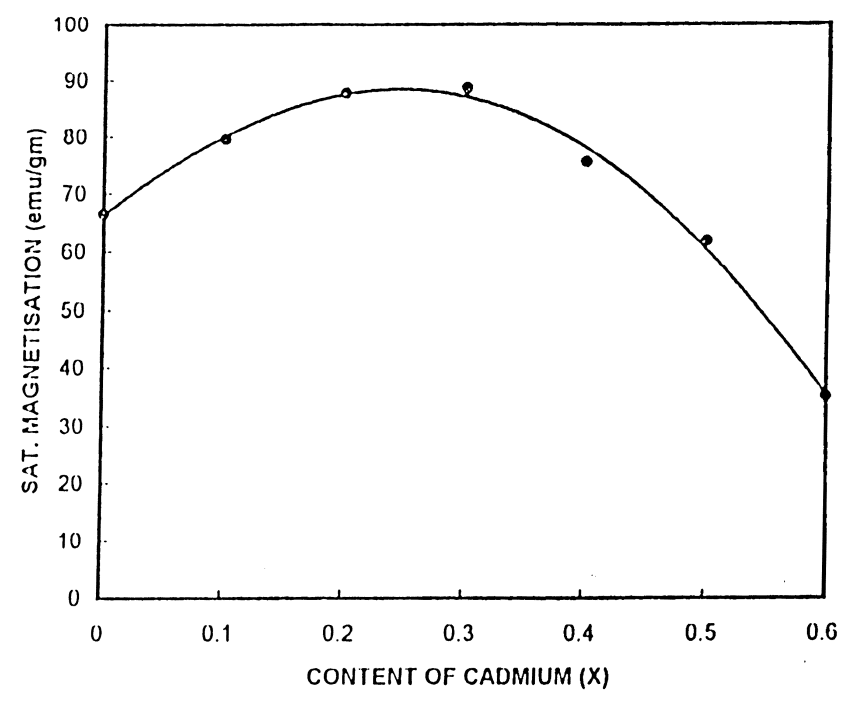

Figure 2. Variation of saturation magnetization with content of cadmium $(x)$.

magnetization of A-sublattice by nonmagnetic $\mathrm{Cd}^{2+}$ ions. This can be explained on the basis of Neel's two sublattice models. The reason for decrease of magnetization beyond $x=0.3$ is that beyond this limit the magnetization of A-sublattice is so much diluted that the A-B interaction becomes weaker than the B-B interaction. This disturbs the parallel arrangement of spin magnetic moments on B-site paving way for canted spins. $\mathrm{Zn}^{2+}$ and $\mathrm{Cd}^{2+}$ substituted ferrites have showed similar type of canting behaviour above a certain limit of their contents.

The compositional variation of initial permeability and anisotropy constant $\left(K_{1}\right)$ with cadmium content is shown in figure 3 . The initial permeability $\left(\mu_{\mathrm{i}}\right)$ increases

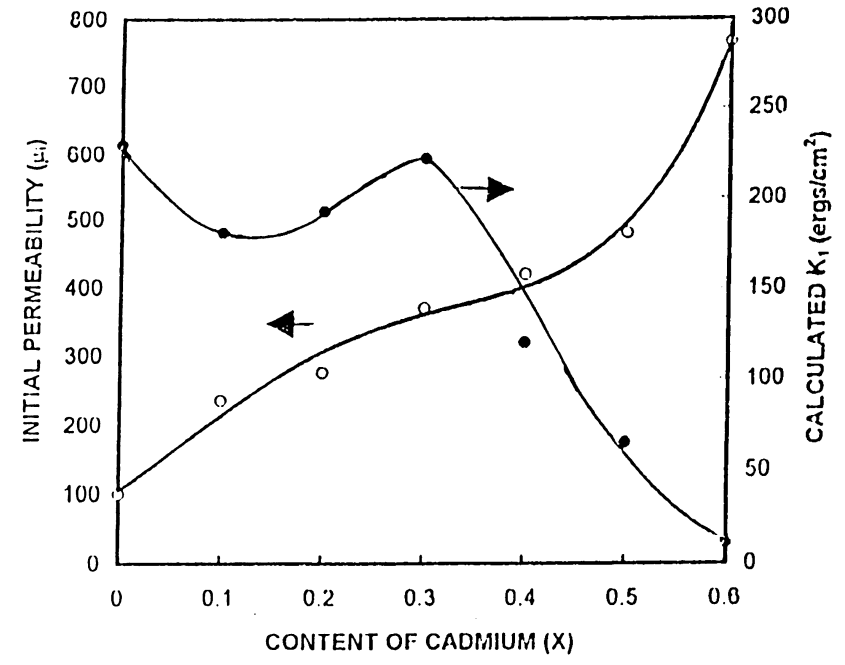

Figure 3. Variation of initial permeability $\left(\mu_{\mathrm{i}}\right)$ and calculated anisotropy constant $\left(K_{1}\right)$ with cadmium content.

continuously with increase in cadmium content up to $x=0 \cdot 6$. The values of $K_{1}$ were calculated using the relation

$$
\mu_{\mathrm{i}}=\frac{M_{\mathrm{s}}^{2} d_{\mathrm{m}}}{K_{1}}
$$

as suggested by Globus (1963), where $d_{\mathrm{m}}$ is the mean grain diameter and $M_{\mathrm{s}}$ the saturation magnetization. The magnetocrystalline anisotropy constant $\left(K_{1}\right)$ shows a continuous decreasing trend. The continuous increase in $\mu_{\mathrm{i}}$ with $x$ can be attributed to rise in both $M_{\mathrm{s}}$ and $d_{\mathrm{m}}$ up to $x=0.3$ and decrease in $K_{1}$ up to $x=0.7$. This is also supported by the fact that the anisotropy constant $\left(K_{1}\right)$ decreases in a rapid manner after this composition.

For low-frequency applications, where a high permeability is required, the crystal anisotropy and the magnetostriction must be as low as possible. It is known that the mobility of domain walls is greatly affected by the microstructure of ferrites. The large grains favour domain wall mobility, giving rise to high permeability and low coercivity. Therefore, the high values of initial permeability in the present samples can also be attributed to the high grain sizes of the samples.

\section{Conclusions}

The lattice parameter increases linearly with increasing cadmium content. Grain size and $M_{\mathrm{s}}$ both increase up to $x=0.3$ and then decrease with further increase in $x$. The initial permeability increases continuously with $x$ and is governed by large grain size and small anisotropy constants $\left(K_{1}\right)$. 


\section{Acknowledgement}

The authors are thankful to Dr S A Patil for extending experimental facilities.

\section{References}

Bellad S S and Chougule B K 1998 Mater. Res. Bull. 33 1165
Bellad S S, Pujar R B and Chougule B K 1998 Mater. Chem. Phys. 52166

Globus A 1963 Ph.D. Thesis, University of Paris, France

Patil R S, Sankpal S V and Sawant S R 1991 Mater. Chem. Phys. 28355

Ravinder D 1994 J. Appl. Phys. 756121

Ravinder D and Seshagiri Rao T 1990 Cryst. Res. Technol. 25 263

Radha K and Ravinder D 1995 Indian J. Pure Appl. Phys. 3374 